\title{
A tighter Cut-Set bound for the multi-terminal erasure channel without side information
}

\author{
Ramin Khalili \\ Supelec-Université Paris XI \\ LSS-CNRS, Paris, France \\ Email: khalili@1ss.supelec.fr
}

\author{
Kavé Salamatian \\ Université Pierre et Marie Curie \\ LIP6-CNRS, Paris, France \\ Email: kave.salamatian@lip6.fr
}

\begin{abstract}
In this paper our recent results in the capacity of single relay erasure channels are used to derive a new and tighter cut-set bound. We initially present a simple and intuitive approach to derive the classical cut-set bound in General Multi-Terminal Erasure Channels. This derivation shows that attaining the bound supposes that a full level of collaboration exists between nodes in the channel. However under some scenarios the full collaboration is not possible. We thereafter use a bounding technique based on reducing every cut-set in a General Multi-Terminal Erasure Channel to a single relay superchannel consisting of super nodes. We show that if a rate setting is not achievable over the single relay super-channel it could not then achieved over the General Multi-Terminal Erasure Channel. This led us to a tighter cut-set type of bound for General MultiTerminal Erasure Channel.
\end{abstract}

\section{INTRODUCTION}

In this paper we assume a class of wireless channels, called erasure channels, where links connecting nodes are erasure channels. In this setting symbols sent over the channel are received errorless or erased and replaced by an erasure symbols $\{e\}$. This is a realistic model for wireless networks as seen by applicative layers if we consider that nodes are not able to use any interference cancellation mechanism, and interferences are suppressed by using separated physical channels, i.e. multiple interface card at nodes. Another way of attaining this separation is through a scheduling mechanism, centralised or decentralized, as in CSMA/CA. In this last setting residual collision, are assumed to be erasures.

In [1] we proposed a derivation for the capacity region of the erasure relay channel without any side information. This setting is the erasure channel counterpart of the classical relay channel as defined in [2]. For this purpose a strong coding converse bound was proved for the single relay erasure channel. This converse bound showed that the classical cut-set bound is not achievable for the single relay erasure channel. The main blocking situation is when the capacity of the sender to relay channel is larger than the capacity of the relay to destination channel and the relay is not able to decode the information sent the source. In this situation a non negligible and non vanishing amount of information should be devoted by the relay to signal to the destination the coding scheme used at relay which restrict the achievable rates to a region smaller than the region predicted by the cut-set bound. Nevertheless, This obstacle has been circumvented in [3] and [4] by adding side information at the receiver. In [3], it is assumed a perfect knowledge of erasure patterns over all erasure channels at receivers and in [4] an in band signaling mechanism is used to transfer the coding scheme used at relay. The results presented in [1] are differentiated from the cited works by the fact that no side information is assumed.

The converse bound was obtained thanks to the nature of the erasure channel that relates directly the amount of information transferred over an erasure channel to the proportion of packets received at receiver. This results in the optimality of Maximal Distance Separable codes for the erasure channel that is the main ingredient of the converse bound.

In this paper we extend the analysis done over a simple one relay erasure channel in [1] to the more general case of multi-sender and multi-receiver. We will first present a new derivation of the general cut-set bound for General MultiTerminal Erasure Channels (GMTEC). This derivation is very simple and intuitive and do not use any information theoretical arguments. However, the obtained bound fit to the classical cut-set bound as derived in [2]. The main contribution of this paper will consist of showing that this cut-set bound is not attainable in general over GMTECs. We will provide a bound tighter than the cut-set bound for these settings. This bound will be derived by transforming the GMTEC to a simple single relay channel obtained by aggregating nodes of the GMTEC into three super-nodes, and applying the capacity region previously obtained for the simple relay channel to this new setting.

\section{General Multi-Terminal ERAsure Channel}

We consider a general multi-terminal erasure channel consisting of a set of $N$ nodes $\mathcal{N}=\{1, . ., N\}$ communicating over a general Vector Erasure Channel ${ }^{1}$. Each node $i$ at the $k$-th transmission time have an associated transmitted variable $X_{i}^{k} \in \mathcal{X}$ and an associated received vector variable $\mathbf{Y}_{i}^{k}=$ $\left(Y_{1 i}^{k}, \ldots, Y_{N i}^{k}\right), Y_{j i}^{k} \in \mathcal{X} \cup\{\mathrm{e}\}$ being the symbol received at node $i$ from node $j$ at the $k^{\text {th }}$ transmission.

In general settings a vector erasure channel could be characterized through a probability measure $\mathbb{P}^{k}=$ $\mathbb{P r o b}\left\{\left(Y_{i j}, i, j \in \mathcal{N}\right) \mid\left(X_{i}, i \in \mathcal{N}\right)\right\}$. This last function gives

\footnotetext{
${ }^{1}$ We use the subscripts to denote node index and superscripts to denote time; bold symbols represent vectors; capitals denote random variables and lower cases denote values.
} 


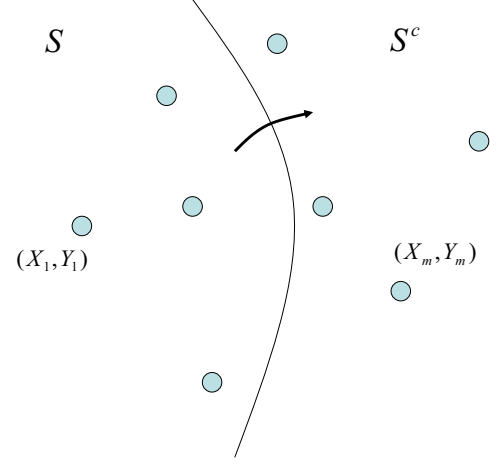

Fig. 1. General multi-terminal network

the joint probability that node $j$ receives from node $i$ a symbol $Y_{i j}^{k}$ if node $i \in \mathcal{N}$ sends $\left\{X_{i}^{k}\right\}$. The erasure nature of the channel and the fact that symbols sent over the channel are not interfering implies that $Y_{i j}$ could be equal to $X_{i}$ or be an erasure, i.e. $y_{i j}=$ e. This general model is very relevant and applicable to computer networks where the received symbols separation is obtained through using different physical channel (interface card) or through a time-sharing mechanism (that could be centralized or distributed mechanism as CSMA/CA access control).

For the special case of non-correlated erasure channel where erasure are not spatially correlated, and under the hypothesis that erasure probability do not depend on the sent symbol $x$, the channel could be simply characterized by an erasure probability matrix $\mathbb{P}^{k}=\left(p_{i j}^{k}\right)_{N \times N} \cdot p_{i j}^{k}$ is the erasure probability of a transmission between nodes $i$ and $j$ at the $k$-th transmission, i.e. $p_{i j}^{k}=\operatorname{Prob}\left\{Y_{i j}^{k}=\mathrm{e}\right\}$. If there is not direct one hop connectivity between two nodes in the channel the packet erasure probability between them is set to one. We might for stationary and ergodic erasure channels drop the superscript $k$ without loss of generality (see [5] for more details). All the subsequent developments are therefore not dependent on any memoryless assumption, i.e. all proofs could be readily extended to the stationary ergodic case by replacing the memoryless erasure probability with the mean probability calculated over the channel statistics.

Let's suppose that corresponding to each transmitterreceiver node pair is a message $W_{i j}$. All messages $W_{i j}$ are assumed independent and uniformly distributed over their respective ranges $\left\{1,2, \ldots, 2^{n R_{i j}}\right\}$. A coding scheme of block length $n$ consists of a set of encoding and decoding mappings, one per node:

- Encoders: The encoder at node $i$ maps the received symbol vectors in the previous $k-1$ transmissions and its own messages to a symbol $X_{i}^{k}$ sent at the $k$-th transmission, i.e. $X_{i}^{k}=f_{i}\left(W_{i 1}, \ldots, W_{i N}, \mathbf{Y}_{i}^{1}, \ldots, \mathbf{Y}_{i}^{k-1}\right), k=$ $1, \ldots, n . \mathbf{X}_{i}^{n}=\left(X_{i}^{k}\right)_{k=1}^{n}$ is the block code of length $n$ sent by node $i$.

- Decoders: The decoders at node $j$ maps the received symbols in each block and its own messages to an estimation of the messages intended to him. In the other term we have $\hat{W}_{i j}=g_{i j}\left(\left(\mathbf{Y}_{i}^{1}, \ldots, \mathbf{Y}_{i}^{n}\right) ;\left(W_{j 1}, \ldots, W_{j N}\right)\right), i \in \mathcal{N}$.
We assume that nodes in $\mathcal{N}$ are divided into two sets, a cut set $\mathcal{S}$ and its complement $\mathcal{S}^{c}$. We define the set of symbols sent by nodes in $\mathcal{A}$ at time $k$ (resp. from time 1 to $k-1$ ) :

$$
\mathcal{X}_{\mathcal{A}}^{k}=\left\{X_{i}^{k} ; i \in \mathcal{A}\right\}, \mathcal{X}_{\mathcal{A}}^{1:(k-1)}=\bigcup_{l=1}^{(k-1)} \mathcal{X}_{\mathcal{A}}^{k}
$$

The set of all symbols sent at time $k$ (resp. from time 1 to $(k-1))$ is defined as :

$$
\mathcal{X}^{k}=\mathcal{X}_{\mathcal{S}}^{k} \cup \mathcal{X}_{\mathcal{S}^{c}}^{k}, \quad \mathcal{X}^{1:(k-1)}=\mathcal{X}_{\mathcal{S}}^{1:(k-1)} \bigcup \mathcal{X}_{\mathcal{S}^{c}}^{1:(k-1)}
$$

Similarly we can define the set of symbols sent at time $k$ (resp. from time 1 to $(k-1)$ ) by nodes in the set $\mathcal{A}$ and received by nodes in $\mathcal{B}$ as :

$$
\mathcal{Y}_{\mathcal{A}, \mathcal{B}}^{k}=\left\{Y_{i j}^{k} ; i \in \mathcal{A} j \in \mathcal{B}\right\}, \mathcal{Y}_{\mathcal{A}, \mathcal{B}}^{1:(k-1)}=\bigcup_{l=1}^{(k-1)} \mathcal{Y}_{\mathcal{A}, \mathcal{B}}^{k}
$$

When the set contains $\mathcal{A}$ (resp. $\mathcal{B}$ ) contains only one node $j$ we will replace the set by the node itself (writing for example $\mathcal{Y}_{j, \mathcal{B}}^{k}$ in place of $\mathcal{Y}_{\{j\}, \mathcal{B}}^{k}$ ).

The classical cut-set bound for the general stationary ergodic non-correlated erasure channel is given in the following lemma :

\section{Lemma 1 (Classical Cut-set bound for erasure channel)}

Let's consider a general non-correlated erasure channel consisting of $N$ nodes characterized by an erasure probability matrix $\mathbb{P}$. If the information rates $\left\{R_{i j}\right\}$ are achievable over this channel then for any subset $S \subset\{1,2, \ldots, N\}$ we have :

$$
\sum_{i \in \mathcal{S}, j \in \mathcal{S}^{c}} R_{i j} \leq \sum_{i \in \mathcal{S}} R_{B i}
$$

where, the broadcast information transfer rate of node $i$ in $\mathcal{S}$ is defined as :

$$
R_{B i}=1-\prod_{j \in \mathcal{S}^{c}} p_{i j}
$$

PROOF Let's start with the simplest case where the cut set $\mathcal{S}$ contains only one node $h$ that have something to broadcast to other nodes. Let's suppose that a code of length $n$ achieves the given rates by sending a block $\mathbf{X}_{h}^{n}$. Under this hypothesis the probability that a symbol $X_{h}^{k}$ is received by a particular node $j$ in $\mathcal{S}^{c}$ is given by $1-p_{h j}$. Respectively, the probability that a symbol $X_{h}^{k}$ is received at least by one node in $\mathcal{S}^{c}$ is $P\left(X_{h}^{k} \in \mathcal{Y}_{h, \mathcal{S}^{c}}^{k}\right)$. From the properties of probability measure functions we have :

$$
\begin{aligned}
P\left(X_{h}^{k} \in \mathcal{Y}_{h, \mathcal{S}^{c}}^{k}\right) & =P\left(X_{h}^{k} \in \bigcup_{j \in \mathcal{S}^{c}} \mathcal{Y}_{h, j}^{k}\right) \\
& =\sum_{i=1}^{\left|\mathcal{S}^{c}\right|}(-1)^{i-1} \sum_{I \subseteq \mathcal{S}^{c} ;|I|=i} P\left(\bigcap_{j \in I} \mathcal{Y}_{h, j}^{n}\right)
\end{aligned}
$$

Moreover we have assumed the channel to be uncorrelated. This leads us to :

$$
P\left(\bigcap_{j \in I} \mathcal{Y}_{h, j}^{n}\right)=\prod_{j \in I}\left(1-p_{h j}\right) .
$$


By replacing in the first equation :

$$
P\left(X_{h}^{k} \in \mathcal{Y}_{h, \mathcal{S}^{c}}^{k}\right)=1-\prod_{j \in \mathcal{S}^{c}} p_{h j}
$$

Using the stationary and ergodic property of the general erasure channel asymptotically for large $n$, the information rate transferred from node $h$ to $\mathcal{S}^{c}$ can not be larger than :

$$
\sum_{j \in \mathcal{S}^{c}} R_{h j}=\frac{1}{n}\left|\mathcal{Y}_{h, \mathcal{S}^{c}}^{k}\right| \leq\left(1-\prod_{j \in \mathcal{S}^{c}} p_{h j}\right)+\epsilon_{n} .
$$

Therefore, for this single sender scenario, the information transfer rate is bounded by :

$$
R_{B h}=\sum_{j \in \mathcal{S}^{c}} R_{h j} \leq 1-\prod_{j \in \mathcal{S}^{c}} p_{h j} .
$$

Now let's go further and consider the general case of multiterminal network of senders and receivers as shown in figure 1. In this setting each sender-receiver pair has a potential message to exchange. We assume that each node in a cut set $\mathcal{S}$ sends a block of $n$ symbols over the channel. For this more general scenario, $\mathcal{E}_{j, \mathcal{S}^{c}}^{k}=\left\{X_{j}^{k} \in \mathcal{Y}_{j, \mathcal{S}^{c}}^{k}\right\}$ denotes the event that symbol $X_{j}^{k}$ sent by node $j$ at time $k$ is received by at least one node in $\mathcal{S}^{c}$. The probability that a symbols sent by one of the nodes in $\mathcal{S}$ is received by at least one node in $\mathcal{S}^{c}$ can be derived as follow :

$$
\begin{aligned}
P\left(\bigcup_{j \in \mathcal{S}} \mathcal{E}_{j, \mathcal{S}^{c}}^{k}\right)= & \sum_{j \in \mathcal{S}} P\left(X_{j}^{k} \in \mathcal{Y}_{j, \mathcal{S}^{c}}^{k}\right)- \\
& \sum_{h=2}^{|\mathcal{S}|}(-1)^{h} \sum_{I \subseteq \mathcal{S} ;|I|=h} P\left(\bigcap_{i \in I} \mathcal{E}_{i, \mathcal{S}^{c}}^{k}\right) \\
\leq & \sum_{j \in \mathcal{S}} P\left(X_{j}^{k} \in \mathcal{Y}_{j, \mathcal{S}^{c}}^{k}\right) \\
\leq & \sum_{j \in \mathcal{S}} R_{B j}
\end{aligned}
$$

where (a) follows from the fact that

$$
\sum_{h=2}^{|\mathcal{S}|}(-1)^{h} \sum_{I \subseteq \mathcal{S} ;|I|=h} P\left(\bigcap_{i \in I} \mathcal{E}_{i, \mathcal{S}^{c}}^{k}\right) \geq 0
$$

and (b) comes from derivation made for the single sender case.

Over a block of size $n, n|\mathcal{S}|$ symbols are sent by senders in $\mathcal{S}$, and asymptotically with large $n$ no more than $n|\mathcal{S}| P\left(\bigcup_{j \in \mathcal{S}} \mathcal{Y}_{s^{c}, j}^{k}\right)+\epsilon_{n}$ different symbols will be received in $\mathcal{S}^{c}$. The rate of information that can be transfered by the channel from $\mathcal{S}$ to $\mathcal{S}^{c}$ is therefore bounded by :

$$
\sum_{i \in \mathcal{S}, j \in \mathcal{S}^{c}} R_{i j} \leq \sum_{i \in \mathcal{S}} R_{i}^{B}
$$

The obtained bounds is a non trivial rewriting of the general and well known cut-set bound [2] in the context of the erasure channels.

Similar bounds have also been derived in [4], [3], however we believe this derivation to be more simple and intuitive. In particular an inspection of the derivation shows that for attaining the bound, one needs to ensure that all the $n \sum_{i \in \mathcal{S}, j \in \mathcal{S}^{c}} R_{i j}$ symbols received at $\mathcal{S}^{c}$ can be combined successfully to decode the sent messages and in particular that no duplicate symbols are generated by nodes in $\mathcal{S}$. In other word, the bound is tight if

$$
\sum_{h=2}^{|\mathcal{S}|}(-1)^{h} \sum_{I \subseteq \mathcal{S} ;|I|=h} P\left(\bigcap_{i \in I} \mathcal{E}_{i, \mathcal{S}^{c}}^{k}\right)=0
$$

For satisfying this condition a high level of collaboration between nodes in the channel is needed. However, under some situations this collaboration is not possible. The essence of the converse bound presented in [1] is in finding these situations.

\section{TIGHT CUT-SET BOUND FOR THE MULTI-TERMINAL ERASURE CHANNEL}

Before getting to the general case of GMTEC, let's start with the simple scenario of single-sender single-relay case analyzed in [1]. In this scenario we have three nodes: one node $(S)$ acting as a sender, one relay node $(R)$ receiving information from sender and collaborating with it to transmit information to receiver through forwarding encoded symbols and one destination node $(D)$ that receives symbols from sender and relay and jointly decode them to figure out the message sent by the sender. This relay erasure channel is characterized by three erasures probabilities : $p_{S R}, p_{S D}, p_{R D}$.

The following converse bound has been presented in [1] :

Theorem 1 (Converse theorem for single relay erasure channel) No deterministic coding scheme can exceed the following bound over an erasure relay channel without side information :

$$
\begin{aligned}
& \left\{\begin{array}{lll}
R<1-p_{S D} p_{S R}, & \text { if }\left(1-p_{S R}\right) \leq\left(1-p_{R D}\right) \\
R<\max \left\{T,\left(1-p_{S D}\right)\right\}, & \text { if }\left(1-p_{S R}\right)>\left(1-p_{R D}\right)
\end{array}\right. \\
& \text { with } T=\min \left\{\left(1-p_{S R}\right),\left(1-p_{S D}\right)+\left(1-p_{R D}\right)\right\}
\end{aligned}
$$

By deterministic coding scheme, we mean a coding scheme such that when given a set of input symbols it will generate a unique output symbol.

A close analysis of the converse bound and a comparison with the general cut-set bound obtained in lemma 1 shows that if $\left.1-p_{S R}\right) \leq\left(1-p_{R D}\right)$, i.e. the capacity of the source to relay channel is smaller than the capacity of the relay to destination channel, the cut-set bound and the converse bound coincides. However when $\left(1-p_{S R}\right)>\left(1-p_{R D}\right)$ the cut-set bound can not be reached. As we proved in [1] this happens because no deterministic coding scheme is able to transfer over the relay to destination channel enough information to reduce at receiver the remaining ambiguity in the symbols received from source. The converse bound suggest two collaboration schemes at the relay : active, passive and no collaboration schemes. Active collaboration is possible when the relay can decode the message sent by sender. We have called this situation the virtually degraded situation. This type of collaboration is possible only when $R<\left(1-p_{S R}\right)$. In the passive collaboration scheme the relay cannot decode the message sent by the source, but nevertheless it can forward "all" its received 


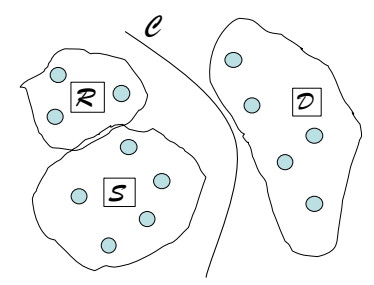

(a) Gathering nodes to super-nodes

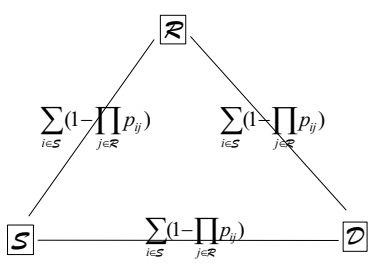

(b) Super relay channel
Fig. 2. Reduction of the multi-terminal problem to the super relay problem

symbols to the destination. This type of collaboration can be applied only when $\left(1-p_{S R}\right) \leq\left(1-p_{R D}\right)$ as the relay to destination channel will be able to send all information at relay. Under situations where $\left(1-p_{S R}\right)>\left(1-p_{R D}\right)$ and $R>\left(1-p_{S R}\right)$ no one of these scheme are useful and the relay cannot ensure any fruitful collaboration with source. In this last situation the cut-set bound is not achievable. It is noteworthy that the well known degraded situation is just a sub case of the virtually degraded situation where the channel is such that each symbols received at destination is guaranteed to have been received at relay.

In the forthcoming we generalize the analysis presented for the single relay channel to the multi-terminal erasure channel. The main idea for tightening the cut-set bound consists of accounting for channel situations where relay nodes have to be in the passively collaborating or non collaborating situation. The bounding technique consists of reducing any particular cut-set choice in a multi-terminal scenario to a particular super-relay channel with a single super-sender node, a single super-relay node and a super-destination. The tighter cut-set bound is obtained by showing that the converse bound on this super-relay channel is in fact an upper bound for the multiterminal channel.

In general settings any node in a multi-terminal network might act in three different roles: as a sender that have some information to transmit, as a relay that receives information from the channel and forward it after encoding or as a receiver that receives information but do not forward it.

Clearly a node might act simultaneously in anyone of these roles or play just one of them. Moreover, based on what we have described for the single relay case, a relay node can be in one of the two following situations :

- Actively collaborating relay : relay nodes that participate in the relaying operations and that validate the virtually degraded assumption toward all subset $\mathcal{A} \subset \mathcal{N}$ containing senders. A node $j$ can be actively collaborating if $R<\sum_{i \in \mathcal{N}}\left(1-p_{i j}\right)$.

- Passively collaborating nodes : nodes participating in relaying but without full access to messages sent by all senders.

Being in one of these situations depends on the channel situation as well as the coding scheme.

Let's assume a cut-set $\mathcal{S}$, partitioning the set of nodes $\mathcal{N}$. We will extract from the set of nodes $\mathcal{N}$ three non-intersecting sets $\mathbb{S}(\mathcal{S}), \mathbb{R}(\mathcal{S})$ and $\mathbb{D}(\mathcal{S})$ and gather all nodes in each one of these sets into a super-node. The super-node $\mathbb{S}(\mathcal{S})$ contains all nodes in $\mathcal{S}$ that act as a source, i.e. have some information to send to other nodes. The super-node $\mathbb{R}(\mathcal{S})$ contains all nodes in $\mathcal{S}$ that act only as relay. Finally we gather in the super-node $\mathbb{D}(\mathcal{S})$ all nodes in $\mathcal{S}^{c}$. In fact by this construction nodes in $\mathcal{S}$ that act only as receiver will not be accounted. This is not a problem as these nodes do not participate in the information transfer from $\mathcal{S}$ to $\mathcal{S}^{c}$.

Now we are assuming that all nodes inside a super-node have access to the each others information, i.e. all senders in $\mathbb{S}$ know what is sent by other senders and all relay in $\mathbb{R}$ know what have been received and sent by other relays. This hypothesis might be seen as unrealistic and very strong. However its use is valid for giving an upper bound for the performance of a realistic communication scheme that will not ensured such a strong information sharing. Using this assumption we might assume that the three super-node $\mathbb{S}, \mathbb{R}$ and $\mathbb{D}$ are connected through three channels (see figure 2(b)) :

- the channel $\mathbb{S} \rightarrow \mathbb{R}$ is an erasure channel with capacity $\sum_{i \in \mathbb{S}}\left(1-\prod_{j \in \mathbb{R}} p_{i j}\right)$. This capacity represents the achievable capacity by directly broadcast of sources without using the relays,

- the channel $\mathbb{S} \rightarrow \mathbb{D}$ is an erasure channel with capacity $\sum_{i \in \mathbb{S}}\left(1-\prod_{j \in \mathbb{D}} p_{i j}\right)$. This capacity represents the available capacity for transferring information needed for relays collaboration with senders

- the channel $\mathbb{R} \rightarrow \mathbb{D}$ is an erasure channel with capacity $\sum_{i \in \mathbb{R}}\left(1-\prod_{j \in \mathbb{D}} p_{i j}\right)$. This capacity represents the available capacity for relays to collaborate with senders.

Each rate assignment over the general multi-terminal problem can be translated to a rate assignment over the described relay channel. Moreover by implementing any achievable rate assignment over the general multi-terminal network, this rate assignment becomes achievable over the super-relay channel. Nevertheless an achievable rate assignment over the superrelay channel will not be achievable over the multi-terminal channel unless one provides mechanisms for full information sharing inside the super-node as assumed in the super-relay channel. The main claim here is that if a rate assignment is not achievable over the super relay channel it would not be achievable over the multi-terminal settings. This results from the fact that the full information sharing in super-node cannot harm the transmission rate and if a rate assignment is not attainable with access to these side information it will not be attained without it.

This leads us to the following theorem.

Theorem 2 Let's consider a general non-correlated erasure channel consisting of $N$ nodes characterized by an erasure probability matrix $\mathbb{P}$. If the information rates $\left\{R_{i j}\right\}$ are achievable over this channel for a fixed coding scheme then for any subset $S \subset\{1,2, \ldots, N\}$ we have :

$$
\text { - For } \begin{aligned}
\sum_{i \in \mathbb{S}} R_{i \mathbb{R}}^{B} \leq & \sum_{i \in \mathbb{R}} R_{i \mathbb{D}}^{B} \\
& \sum_{i \in \mathcal{S}, j \in \mathcal{S}^{c}} R_{i j}<\sum_{i \in \mathbb{S}} R_{i(\mathbb{R} \cup \mathbb{D})}^{B}
\end{aligned}
$$




$$
\begin{aligned}
\text { - For } \sum_{i \in \mathbb{S}} R_{i \mathbb{R}}^{B}>\sum_{i \in \mathbb{R}} R_{i \mathbb{R}}^{B} \\
\sum_{i \in \mathcal{S}, j \in \mathcal{S}^{c}} R_{i j}<\max \left\{T, \sum_{i \in \mathbb{S}} R_{i \mathbb{D}}^{B}\right\}
\end{aligned}
$$

with $T=\min \left\{\sum_{i \in \mathbb{S}} R_{i \mathbb{R}}^{B}, \sum_{i \in \mathcal{S}} R_{i \mathbb{D}}^{B}\right\}$

where, the broadcast information transfer rate of node $i$ to the set $\mathcal{A}$ is defined as :

$$
R_{i \mathcal{A}}^{B}=1-\prod_{j \in \mathcal{A}} p_{i j} .
$$

PROOF The proof is based on the described reduction procedure of any multi-terminal setting to a super relay problem. The given bounds are the upper bound derived in the converse theorem for relay erasure channel applied to the super relay relative to a cut-set $\mathcal{S}$.

\section{Remarks}

- A comparison of the tightened cut-set bound with the classical cut-set bound shows some differences. For the case where $\sum_{i \in \mathbb{S}} R_{i \mathbb{R}}^{B} \leq \sum_{i \in \mathbb{R}} R_{i \mathbb{D}}^{B}$, if the transmission rate $\sum_{i \in \mathcal{S}, j \in \mathcal{S}^{c}} R_{i j}$ exceeds $\sum_{i \in \mathbb{S}} R_{i \mathbb{R}}^{B}$ active cooperation is not possible for some of the relay nodes in $\mathbb{R}$ and the attained rate is lower than what predicted by the classical cut-set bound. For the case where $\sum_{i \in \mathbb{S}} R_{i \mathbb{R}}^{B}>\sum_{i \in \mathbb{R}} R_{i \mathbb{D}}^{B}$, cut-set bound is attainable only when $\sum_{i \in \mathcal{S}, j \in \mathcal{S}^{c}} R_{i j} \leq \sum_{i \in \mathbb{S}} R_{i \mathbb{R}}^{B}$, i.e. under virtually degraded condition.

- Nodes in actively collaborating situation can decode all the messages sent by the senders. This means that they are equivalent to senders that have also access to all sent information. They can therefore be integrated to the set $\mathbb{S}$. This will tighten the previous bound for specific coding schemes where such nodes exists. By doing this the set of relays $\mathbb{R}$ contains only nodes in the "passively collaborating" situations.

- As explained previously having access to side information remove the restriction that we had on cut-set bound achievability for single relay channels. It appears that in general settings the classical cut-set bound should be reachable with access to side informations. One approach proposed in [3] uses the sequence of all losses incurred during the transmission over all links in the final receiver as a side-information and reach the cut-set bound. Nevertheless, the amount of extra information needed to transfer the side information should be assessed. A simple evaluation shows that one needs $n\left(1-h\left(p_{S R}\right)\right)$ bits of extra information to transfer the side information which is the erasure pattern over the sender to relay channel. Accounting the information rate needed for side information the scheme proposed in [3] attains a proportion $\frac{\mathbf{R} \log (|\mathcal{X}|)}{\mathbf{R} \log (|\mathcal{X}|)+\left(1-h\left(p_{S R}\right)\right)}$ of the cut-set bound. Moreover, no practical means of reliably transferring this side information is provided.

- Another solution to expand the converse bound is proposed in [4]. Classically in information theory, one chooses a random code at the beginning of the communication and informs the receiver about the used coding scheme. The used coding scheme might be seen as side information that is given at the beginning of the communication. However, in [4], the use of a randomly changing coding scheme is proposed, where the relay node chooses randomly at each transmission how to mix the received symbols (as in classical settings) but changes this mixture randomly during the transmission. A naive evaluation of the amount of extra information needed to transfer the side information leads to $\mathcal{O}(n)$ bits per transferred packet (this information placed in the packet headers). By choosing the packet lengths enough large the over head fraction per each packet can be made arbitrarily small. In practice, the packet length can not be make arbitrary large and the overhead could be really significant specially for large size networks.

\section{CONCLUSION}

We derived the classical cut-set bound for the erasure channels and we showed that this bound is not achievable in general. The main reason is that attaining the bound supposes that a full level of collaboration exists between nodes in the channel. It might be happen only if the channel is in the degraded situation, while under some scenarios the full collaboration is not possible. We defined three types of the node collaboration in the channel : active, passive and without collaboration.Based on this precise analysis we are able to find the optimum encoding scheme for nodes in the channel which leads us to a tighter upper bound for the capacity region of the erasure channel.

We believe the bounding technique to be also useful in the more general case of channel with error links to make the classical cut-set bound tighter.

\section{REFERENCES}

[1] K. Salamatian and R. Khalili, "An information theory for erasure channels (invited paper)," Allerton, 2005

[2] T. M. Cover and J. A. Thomas, Elements of Information Theory. John Wiley Sons, New York, 1991.

[3] R. Gowaikar, A. F. Dana, R. Palanki, B. Hassibi, and M. Effros, "On the capacity of wireless erasure networks," "ISIT, 2004.

[4] D. S. Lun, M. Mdard, and M. Effros, "On coding for reliable communication over packet networks," Allerton, 2004.

[5] R. Khalili and K. Salamatian, "An informatin theory of erasure channels," tech. rep., 2005. 